\title{
A Tauberian theorem for the statistical generalized Nörlund-Euler summability method
}

\author{
Naim L. Braha \\ Ilirias research Institute Kosovo and \\ Department of Mathematics and the Computer \\ Sciences,University of Prishtina, Kosovo \\ email: nbraha@yahoo.com
}

Abstract. Let $\left(p_{n}\right)$ and $\left(q_{n}\right)$ be any two non-negative real sequences with

$$
R_{n}:=\sum_{k=0}^{n} p_{k} q_{n-k} \neq 0 \quad(n \in \mathbb{N}) .
$$

With $E_{n}^{1}$ - we will denote the Euler summability method. Let $\left(x_{n}\right)$ be a sequence of real or complex numbers and set

$$
N_{p, q}^{n} E_{n}^{1}:=\frac{1}{R_{n}} \sum_{k=0}^{n} p_{k} q_{n-k} \frac{1}{2^{k}} \sum_{v=0}^{k}\left(\begin{array}{l}
k \\
v
\end{array}\right) x_{v}
$$

for $n \in \mathbb{N}$. In this paper, we present necessary and sufficient conditions under which the existence of the st- limit of $\left(x_{n}\right)$ follows from that of $s t-N_{p, q}^{n} E_{n}^{1}-$ limit of $\left(x_{n}\right)$. These conditions are one-sided or two-sided if $\left(x_{n}\right)$ is a sequence of real or complex numbers, respectively.

\section{Introduction}

In what follows we give the concept of the summability method known as the generalized Nörlund summability method $(N, p, q)$ (see [1]). Given two

2010 Mathematics Subject Classification: 40G15, 41A36

Key words and phrases: generalized Nörlund-Euler summability; one-sided and two-sided Tauberian conditions, statistical convergence 
non-negative sequences $\left(p_{n}\right)$ and $\left(q_{n}\right)$, the convolution $(p \star q)$ is defined by

$$
R_{n}:=(p \star q)_{n}=\sum_{k=0}^{n} p_{k} q_{n-k}=\sum_{k=0}^{n} p_{n-k} q_{k}
$$

In this paper we suppose $R_{n} \rightarrow \infty$ as $n \rightarrow \infty$. With $E_{n}^{1}-$ we will denote the Euler summability method. Let $\left(x_{n}\right)$ be a sequence. When $(p \star q)_{n} \neq 0$ for all $n \in \mathbb{N}$, the generalized Nörlund-Euler transform of the sequence $\left(x_{n}\right)$ is the sequence $N_{p, q}^{n} E_{n}^{1}$ obtained by putting

$$
N_{p, q}^{n} E_{n}^{1}=\frac{1}{(p \star q)_{n}} \sum_{k=0}^{n} p_{k} q_{n-k} \frac{1}{2^{k}} \sum_{v=0}^{k}\left(\begin{array}{l}
k \\
v
\end{array}\right) x_{v}
$$

We say that the sequence $\left(x_{n}\right)$ is generalized Nörlund-Euler summable to L determined by the sequences $\left(p_{n}\right)$ and $\left(q_{n}\right)$ or briefly summable $N_{p, q}^{n} E_{n}^{1}$ to $L$ if

$$
\lim _{n \rightarrow \infty} N_{p, q}^{n} E_{n}^{1}=L \text {. }
$$

Suppose throughout the paper we assume that the sequence $q=\left(q_{n}\right)$ satisfies the following conditions:

$$
\begin{aligned}
q_{t_{n}-k} & \leq 2 q_{n-k}, k=0,1,2,3, \cdots, n ; t>1, \\
q_{n-k} & \leq 2 q_{t_{n-k}}, k=0,1,2,3, \cdots, t_{n} ; 0<t<1,
\end{aligned}
$$

where $t_{n}=[t \cdot n]$.

If

$$
\lim _{n \rightarrow \infty} x_{n}=L
$$

implies (2), then the summation method generated by $N_{p, q}^{n} E_{n}^{1}$ is regular, and it is satisfied under certain conditions. However, the converse is not always true. We can show by the following example

Example 1 Let us consider that $\mathrm{x}=\left(\mathrm{x}_{\mathrm{k}}\right)=(-1)^{\mathrm{k}}$, then we have

$$
\left|\frac{1}{R_{n}} \sum_{k=0}^{n} p_{k} q_{n-k} \frac{1}{2^{k}} \sum_{v=0}^{k}\left(\begin{array}{l}
k \\
v
\end{array}\right)(-1)^{v}\right| \leq \frac{1}{R_{n}} \sum_{k=0}^{n} p_{k} q_{n-k} \frac{1}{2^{k}} \sum_{v=0}^{k}\left(\begin{array}{l}
k \\
v
\end{array}\right) 1 \rightarrow 1 \text { as } n \rightarrow \infty .
$$

And as we know $\mathrm{x}=\left(\mathrm{x}_{\mathrm{k}}\right)$, is not convergent. 
Notice that (2) may imply (5) under a certain condition, which is called a Tauberian condition. Any theorem which states that convergence of a sequence follows from its $N_{p, q}^{n} E_{n}^{1}$ summability and some Tauberian condition is said to be a Tauberian theorem for the $\mathrm{N}_{p, q}^{n} E_{n}^{1}$ summability method. The inclusion and Tauberian type theorems are proved in the papers $[6,7,2,3,4,5,8]$, and some theorems of inclusion, Tauberian and convexity type for certain families of generalized Nörlund methods are obtained in [9].

\section{Results}

In this paper, we present necessary and sufficient conditions under which the existence of the limit $s t-\lim _{n \rightarrow \infty} x_{n}=$ L follows from that of $s t-$ $\lim _{n \rightarrow \infty} N_{p, q}^{n} E_{n}^{1}=$ L. These conditions are one-sided or two-sided if $\left(x_{n}\right)$ is a sequence of real or complex numbers, respectively.

Definition 1 A sequence $\left(x_{n}\right)$ is statistically convergent to $\mathrm{L}$, if for every $\epsilon>0$, we have

$$
\lim _{n \rightarrow \infty} \frac{\left|\left\{k \leq n:\left|x_{k}-L\right| \geq \epsilon\right\}\right|}{n}=0,
$$

where $|A|$, stands for cardinality of the set.

The theory of Tauberian is extensively studied by many authors ([1], [2], [3], [4], [7], [9]). In this section our aim is to find conditions (so-called Tauberian) under which the converse implication holds, for defined convergence. Exactly, we will prove under which conditions convergence of sequences $\left(x_{n}\right)$, follows from $N_{p, q}^{n} E_{n}^{1}-$ convergence.

Definition 2 A sequence $\left(\mathrm{x}_{\mathfrak{n}}\right)$ is weighted $\mathrm{N}_{\mathrm{p}, \mathrm{q}}^{\mathrm{n}} \mathrm{E}_{\mathfrak{n}}^{1}$-statistically convergent to $\mathrm{L}$ if for every $\epsilon>0$,

$$
\lim _{n \rightarrow \infty} \frac{1}{R_{n}}\left|\left\{k \leq R_{n}:\left|\frac{1}{R_{n}} \sum_{k=0}^{n} p_{k} q_{n-k} \frac{1}{2^{k}} \sum_{v=0}^{k}\left(\begin{array}{l}
k \\
v
\end{array}\right) x_{v}-L\right| \geq \epsilon\right\}\right|=0 .
$$

And we say that the sequence $\left(x_{n}\right)$ is statistically summable to $L$ by the weighted summability method $N_{p, q}^{n} E_{n}^{1}$, if $s t-\lim _{n} N_{p, q}^{n} E_{n}^{1}=L$. We denote by $N_{p, q}^{n} E_{n}^{1}(s t)$ the set of all sequences which are statistically summable.

Theorem 1 If sequence $\mathrm{x}=\left(\mathrm{x}_{\mathrm{n}}\right)$ is $\mathrm{N}_{\mathrm{p}, \mathrm{q}}^{\mathrm{n}} \mathrm{E}_{\mathrm{n}}^{1}$ summable to $\mathrm{L}$, then sequence $x=\left(x_{n}\right)$ is $\mathrm{N}_{\mathfrak{p}, q}^{n} \mathrm{E}_{\mathfrak{n}}^{1}-$ statistically convergent to L. But not conversely. 
Proof. The first part of the proof is obvious. To prove the second part we will show this example:

Example 2 Let us consider that $\left(\mathrm{p}_{\mathrm{k}}\right)=(1),\left(\mathrm{q}_{\mathrm{n}-\mathrm{k}}\right)=\left(2^{\mathrm{k}}\right)$, and define

$$
x_{k}= \begin{cases}\sqrt{2^{k}}, & \text { for } k=2^{n} \\ 0, & \text { otherwise }\end{cases}
$$

Under this conditions we get:

$$
\frac{1}{2^{n}-1}\left|\left\{k \leq 2^{n}-1:\left|\frac{1}{2^{n}-1} \sum_{k=0}^{n} 1 \cdot \sum_{v=0}^{k}\left(\begin{array}{l}
k \\
v
\end{array}\right) x_{v}-0\right| \geq \epsilon\right\}\right| \leq \frac{\sqrt{2^{n}}}{2^{n}-1} \rightarrow 0 .
$$

Hence, it is $\mathrm{N}_{\mathrm{p}, \mathrm{q}}^{\mathrm{n}} \mathrm{E}_{\mathrm{n}}^{1}$ statistically summable to 0 . On the other hand, if we take in consideration that $\mathrm{k}=2^{\mathrm{n}}$, we get

$$
\frac{1}{2^{\mathrm{n}}-1} \sum_{\mathrm{k}=0}^{\mathrm{n}} 1 \cdot \sum_{v=0}^{\mathrm{k}}\left(\begin{array}{l}
\mathrm{k} \\
v
\end{array}\right) \mathrm{x}_{v} \rightarrow \infty, \quad \text { as } \mathrm{n} \rightarrow \infty
$$

From last relation follows that $\mathrm{x}=\left(\mathrm{x}_{\mathrm{n}}\right)$ is not $\mathrm{N}_{\mathrm{p}, \mathrm{q}}^{\mathrm{n}} \mathrm{E}_{\mathrm{n}}^{1}$ summable to 0 .

Theorem 2 Let us suppose that sequence $\left(\mathrm{x}_{\mathrm{n}}\right)$-statistically convergent to $\mathrm{L}$, and

$$
\sup _{\substack{0 \leq v \leq k \\ 0 \leq k \leq n \\ n \in \mathbb{N}}}(v+k+n)\left|x_{v}-\mathrm{L}\right|<\infty .
$$

Then it converges $\mathrm{N}_{\mathrm{p}, \mathrm{q}}^{\mathrm{n}} \mathrm{E}_{\mathrm{n}}^{1}$-statistically to L. Converse is not true.

Proof. From fact that $\left(x_{n}\right)$ converges statistically to L, we get

$$
\lim _{n \rightarrow \infty} \frac{\left|\left\{k \leq n:\left|x_{k}-L\right| \geq \epsilon\right\}\right|}{n}=0 .
$$

Let us denote by $B_{\epsilon}=\left\{k \leq n:\left|x_{k}-L\right| \geq \epsilon\right\}$ and $\overline{B_{\epsilon}}=\left\{k \leq n:\left|x_{k}-L\right|<\epsilon\right\}$. Then

$$
\left|\frac{1}{R_{n}} \sum_{k=0}^{n} p_{k} q_{n-k} \frac{1}{2^{k}} \sum_{v=0}^{k}\left(\begin{array}{l}
k \\
v
\end{array}\right) x_{v}-L\right|=\left|\frac{1}{R_{n}} \sum_{k=0}^{n} p_{k} q_{n-k} \frac{1}{2^{k}} \sum_{v=0}^{k}\left(\begin{array}{l}
k \\
v
\end{array}\right)\left(x_{v}-L\right)\right| \leq
$$




$$
\frac{1}{R_{n}} \sum_{\substack{k=0 \\
k \in B_{\epsilon}}}^{n} p_{k} q_{n-k} \frac{1}{2^{k}} \sum_{v=0}^{k}\left(\begin{array}{l}
k \\
v
\end{array}\right)\left|x_{v}-L\right|+\frac{1}{R_{n}} \sum_{\substack{k=0 \\
k \in \bar{B}_{\epsilon}}}^{n} p_{k} q_{n-k} \frac{1}{2^{k}} \sum_{v=0}^{k}\left(\begin{array}{l}
k \\
v
\end{array}\right)\left|x_{v}-L\right| \leq
$$

(from given conditions for sequence $\left(x_{v}\right)$, there exists a constant $C$ such that $\left.\left|x_{v}-\mathrm{L}\right| \leq \frac{\mathrm{C}}{v+\mathrm{k}+\mathrm{n}}\right)$

$$
\begin{gathered}
\frac{C}{R_{n}} \sum_{\substack{k=0 \\
k \in B_{\epsilon}}}^{n} p_{k} q_{n-k} \frac{1}{2^{k}} \sum_{v=0}^{k}\left(\begin{array}{l}
k \\
v
\end{array}\right) \frac{1}{v+k+n}+\frac{\epsilon}{R_{n}} \sum_{\substack{k=0 \\
k \in \bar{B}_{\epsilon}}}^{n} p_{k} q_{n-k} \frac{1}{2^{k}} \sum_{v=0}^{k}\left(\begin{array}{l}
k \\
v
\end{array}\right) \leq \\
\frac{C}{R_{n}} \sum_{\substack{k=0 \\
k \in B_{\epsilon}}}^{n} p_{k} q_{n-k} \frac{1}{2^{k}} \frac{1}{k+n} \sum_{v=0}^{k}\left(\begin{array}{l}
k \\
v
\end{array}\right)+\frac{\epsilon}{R_{n}} \sum_{k=0}^{n} p_{k} q_{n-k} \frac{1}{2^{k}} \sum_{v=0}^{k}\left(\begin{array}{l}
k \\
v
\end{array}\right) \leq \\
\leq \frac{C\left|B_{\epsilon}\right|}{n} \cdot \frac{\max _{0 \leq k \leq n}\left\{p_{k} q_{n-k}\right\}}{R_{n}}+\epsilon \rightarrow 0+\epsilon, \quad \text { as } n \rightarrow \infty .
\end{gathered}
$$

To show that converse is not true we will use into consideration this

Example 3 Let us consider that $\left(\mathrm{p}_{\mathrm{n}}\right)=1,\left(\mathrm{q}_{\mathrm{n}-\mathrm{k}}\right)=\left(2^{\mathrm{k}}\right)$ for $\mathrm{n} \in \mathbb{N}$ and we define the sequence $\mathrm{x}=\left(\mathrm{x}_{\mathfrak{n}}\right)$, as follows:

$$
x_{k}=\left\{\begin{array}{ll}
1, & \text { for } k=m^{2}-m, \cdots, m^{2}-1 \\
-m, & \text { for } k=m^{2}, m=2, \cdots \\
0, & \text { otherwise }
\end{array} .\right.
$$

Under this conditions, after some calculations we get:

$$
\left|\frac{1}{2^{n}-1} \sum_{k=0}^{n} 1 \cdot \sum_{v=0}^{k}\left(\begin{array}{l}
k \\
v
\end{array}\right) x_{v}-1\right| \leq\left|\frac{1}{2^{n}-1} \sum_{k=0}^{n} 1 \cdot \sum_{v=0}^{k}\left(\begin{array}{l}
k \\
v
\end{array}\right)-1\right|=0 .
$$

From last relation follows that $\mathrm{x}=\left(\mathrm{x}_{\mathrm{n}}\right)$ is $\mathrm{N}_{\mathrm{p}, \mathrm{q}}^{\mathrm{n}} \mathrm{E}_{\mathfrak{n}}^{1}-$ summable to 1 . Hence from Theorem 1, $\left(\mathrm{x}_{\mathfrak{n}}\right)$ is $\mathrm{N}_{\mathfrak{p}, \mathfrak{q}}^{n} \mathrm{E}_{\mathfrak{n}}^{1}$ - statistically convergent. On the other hand, the sequence $\left(\mathrm{m}^{2} ; \mathrm{m}=2,3 \cdots,\right)$ has natural density zero and it is clear that st $-\liminf _{\mathfrak{n}} x_{n}=0$ and $s t-\limsup _{n} x_{n}=1$. Thus, $\left(x_{k}\right)$ is not statistically convergent.

\section{Theorem 3 If}

$$
s t-\liminf _{n} \frac{R_{t_{n}}}{R_{n}}>1, t>1
$$


where $\mathrm{t}_{\mathrm{n}}$, denotes the integral parts of the $[\mathrm{tn}]$ for every $\mathrm{n} \in \mathbb{N}$, and let $\left(\mathrm{x}_{\mathrm{k}}\right)$ be a sequence of real numbers which converges to $\mathrm{L}, \mathrm{N}_{\mathrm{p}, \mathrm{q}}^{\mathrm{n}} \mathrm{E}_{\mathrm{n}}^{1}-$ statistically. Then $\left(\mathrm{x}_{\mathrm{k}}\right)$ is st-convergent to the same number $\mathrm{L}$ if and only if the following two conditions hold:

$\inf _{t>1} \limsup _{n} \frac{1}{R_{n}}\left|\left\{k \leq R_{n}: \frac{1}{R_{t_{k}}-R_{k_{j}}} \sum_{j=k+1}^{t_{k}} p_{j} q_{t_{k}-j} \frac{1}{2^{j}} \sum_{v=0}^{j}\left(\begin{array}{l}j \\ v\end{array}\right)\left(x_{v}-x_{k}\right) \leq-\epsilon\right\}\right|=0$

and

$\inf _{0<t<1} \limsup _{n} \frac{1}{R_{n}}\left|\left\{k \leq R_{n}: \frac{1}{R_{k}-R_{t_{k_{j}}=t_{k}+1}} \sum_{j}^{k} p_{j} q_{k-j} \frac{1}{2^{j}} \sum_{v=0}^{j}\left(\begin{array}{l}j \\ v\end{array}\right)\left(x_{k}-x_{v}\right) \leq-\epsilon\right\}\right|=0$.

Remark 1 Let us suppose that st - $\lim _{\mathrm{k}} \mathrm{x}_{\mathrm{k}}=\mathrm{L} ;\left(\mathrm{x}_{\mathrm{n}}\right)$ is $\mathrm{N}_{\mathrm{p}, \mathrm{q}}^{\mathrm{n}} \mathrm{E}_{\mathrm{n}}^{1}-$ statistically convergent and relation (6) satisfies, then for every $\mathrm{t}>1$, is valid the following relation:

$$
s t-\lim _{k} \frac{1}{R_{t_{k}}-R_{k}} \sum_{j=k+1}^{t_{k}} p_{j} q_{t_{k}-j} \frac{1}{2^{j}} \sum_{v=0}^{j}\left(\begin{array}{l}
j \\
v
\end{array}\right)\left(x_{v}-x_{k}\right)=0
$$

and in case where $0<\mathrm{t}<1$,

$$
s t-\lim _{k} \frac{1}{R_{k}-R_{t_{k}}} \sum_{j=t_{k}+1}^{k} p_{j} q_{k-j} \frac{1}{2^{j}} \sum_{v=0}^{j}\left(\begin{array}{l}
j \\
v
\end{array}\right)\left(x_{k}-x_{v}\right)=0 .
$$

In the next result, we will consider the case where $x=\left(x_{n}\right)$ is a sequence of complex numbers.

Theorem 4 Let us suppose that relation (6) is satisfied. And $\left(x_{\mathfrak{n}}\right)$ be a sequence of complex numbers, which is $\mathrm{N}_{\mathrm{p}, \mathrm{q}}^{\mathrm{n}} \mathrm{E}_{\mathrm{n}}^{1}-$ statistically convergent to $\mathrm{L}$. Then $\left(x_{n}\right)$ is st-convergent to the same number $\mathrm{L}$ if and only if the following two conditions hold:

$$
\inf _{t>1} \limsup _{n} \frac{1}{R_{n}}\left|\left\{k \leq R_{n}:\left|\frac{1}{R_{t_{k}}-R_{k}} \sum_{j=k+1}^{t_{k}} p_{j} q_{t_{k}-j} \frac{1}{2^{j}} \sum_{v=0}^{j}\left(\begin{array}{l}
j \\
v
\end{array}\right)\left(x_{v}-x_{k}\right)\right| \geq \epsilon\right\}\right|=0
$$


and

$$
\inf _{0<t<1} \limsup _{n} \frac{1}{R_{n}}\left|\left\{k \leq R_{n}:\left|\frac{1}{R_{k}-R_{t_{k_{j}}=t_{k}+1}} \sum_{j}^{k} p_{j} q_{k-j} \frac{1}{2^{j}} \sum_{v=0}^{j}\left(\begin{array}{l}
j \\
v
\end{array}\right)\left(x_{k}-x_{v}\right)\right| \geq \epsilon\right\}\right|=0 .
$$

In what follows we will show some auxiliary lemmas which are needful in the sequel.

Lemma 1 Condition given by relation (6) is equivalent to this one:

$$
s t-\liminf _{n} \frac{R_{n}}{R_{t_{n}}}>1, \quad 0<t<1 .
$$

Proof. Let us suppose that relation (6) is valid, $0<t<1$ and $m=t_{n}=[t \cdot n]$, $n \in \mathbb{N}$. Then it follows that

$$
\frac{1}{t}>1 \Rightarrow \frac{m}{t}=\frac{[t \cdot n]}{t} \leq n,
$$

from above relation we obtain:

$$
\frac{R_{n}}{R_{t_{n}}} \geq \frac{R_{\left[\frac{m}{t}\right]}}{R_{t_{n}}} \Rightarrow s t-\liminf _{n} \frac{R_{n}}{R_{t_{n}}} \geq s t-\liminf _{n} \frac{R_{\left[\frac{m}{t}\right]}}{R_{t_{n}}}>1 .
$$

Conversely, let us suppose that relation (13) is valid. Let $t>1$ be given number and let $t_{1}$ be chosen such that $1<t_{1}<t$. Set $m=t_{n}=[t \cdot n]$. From $0<\frac{1}{\mathrm{t}}<\frac{1}{\mathrm{t}_{1}}<1$, it follows that:

$$
\mathrm{n} \leq \frac{\mathrm{tn}-1}{\mathrm{t}_{1}}<\frac{[\mathrm{tn}]}{\mathrm{t}_{1}}=\frac{\mathrm{m}}{\mathrm{t}_{1}}
$$

provided $t_{1} \leq t-\frac{1}{n}$, which is a case where if $n$ is large enough. Under this conditions we have:

$$
\frac{R_{t_{n}}}{R_{n}} \geq \frac{R_{t_{n}}}{R_{\left[\frac{m}{t_{1}}\right]}} \Rightarrow s t_{\lambda}-\liminf _{n} \frac{R_{t_{n}}}{R_{n}} \geq s t_{\lambda}-\liminf _{n} \frac{R_{t_{n}}}{R_{\left[\frac{m}{t_{1}}\right]}}>1 .
$$

Lemma 2 Let us suppose that relation (6) is satisfied and let $\mathrm{x}=\left(\mathrm{x}_{\mathrm{k}}\right)$ be a sequence of complex numbers which is $\mathrm{N}_{\mathrm{p}, \mathrm{q}}^{\mathrm{n}} \mathrm{E}_{\mathfrak{n}}^{1}$-statistically convergent to $\mathrm{L}$. Then for every $\mathrm{t}>0$,

$$
s t-\lim _{n} N_{p, q}^{t_{n}} E_{n}^{1}=L .
$$


Proof. (I) Let us consider that $t>1$. Then

$$
\lim _{n \rightarrow \infty} \frac{1}{R_{n}} \sum_{k=0}^{n} p_{k} q_{n-k} \frac{1}{2^{k}} \sum_{v=0}^{k}\left(\begin{array}{l}
k \\
v
\end{array}\right) x_{v}=\lim _{n \rightarrow \infty} \frac{1}{R_{t_{n}}} \sum_{k=0}^{t_{n}} p_{k} q_{t_{n}-k} \frac{1}{2^{k}} \sum_{v=0}^{k}\left(\begin{array}{l}
k \\
v
\end{array}\right) x_{v},
$$

and for every $\epsilon>0$ we have:

$$
\begin{gathered}
\left\{k \leq R_{t_{n}}:\left|N_{p, q}^{t_{n}} E_{n}^{1}-L\right| \geq \epsilon\right\} \subset\left\{k \leq R_{n}:\left|N_{p, q}^{n} E_{n}^{1}-L\right| \geq \epsilon\right\} \cup \\
\left\{k \leq R_{n}: \frac{1}{R_{n}} \sum_{k=0}^{n} p_{k} q_{n-k} \frac{1}{2^{k}} \sum_{v=0}^{k}\left(\begin{array}{l}
k \\
v
\end{array}\right) x_{v} \neq \frac{1}{R_{t_{n}}} \sum_{k=0}^{t_{n}} p_{k} q_{t_{n}-k} \frac{1}{2^{k}} \sum_{v=0}^{k}\left(\begin{array}{l}
k \\
v
\end{array}\right) x_{v}\right\} .
\end{gathered}
$$

Now proof of the lemma in this case follows from relation (14) and st $\lim _{n} N_{p, q}^{n} E_{n}^{1}=L$.

(II) In this case we have that $0<t<1$. For $t_{n}=[t \cdot n]$, for any natural number $n$, we can conclude that $N_{p, q}^{t_{n}} E_{n}^{1}$ does not appears more than $\left[1+t^{-1}\right]$ times in the sequence $N_{p, q}^{n} E_{n}^{1}$. In fact if there exist integers $k, l$ such that

$$
n \leq t \cdot k<t(k+1)<\cdots<t(k+l-1)<n+1 \leq t(k+l),
$$

then

$$
\mathrm{n}+\mathrm{t}(\mathrm{l}-1) \leq \mathrm{t}(\mathrm{k}+\mathrm{l}-1)<\mathrm{n}+1 \Rightarrow \mathrm{l}<1+\frac{1}{\mathrm{t}} .
$$

And we have this estimation

$$
\begin{aligned}
& \frac{1}{R_{n}}\left|\left\{k \leq R_{n}:\left|N_{p, q}^{t_{n}} E_{n}^{1}-L\right| \geq \epsilon\right\}\right| \\
& \leq\left(1+\frac{1}{t}\right) \frac{1}{R_{n}}\left|\left\{k \leq R_{t_{n}}:\left|N_{p, q}^{n} E_{n}^{1}-L\right| \geq \epsilon\right\}\right| \\
& \leq 2(1+t) \frac{1}{R_{t_{n}}}\left|\left\{k \leq R_{t_{n}}:\left|N_{p, q}^{n} E_{n}^{1}-L\right| \geq \epsilon\right\}\right|,
\end{aligned}
$$

provided $\frac{1}{R_{n}}\left(\frac{t+1}{t}\right) \leq 2(t+1) \frac{1}{R_{t_{n}}}$, which is the case where $n$ is large enough. From last relation it follows: $s t-\lim _{n} N_{p, q}^{t_{n}} E_{n}^{1}=L$.

Proposition 1 Let us suppose that relation (6) is satisfied and let $\mathrm{x}=\left(\mathrm{x}_{\mathrm{k}}\right)$ be a sequence of complex numbers which is $\mathrm{N}_{\mathfrak{p}, \mathrm{q}}^{t_{n}} \mathrm{E}_{\mathfrak{n}}^{1}$-statistically convergent to L. Then for every $\mathrm{t}>1$,

$$
s t-\lim _{k} \frac{1}{R_{t_{k}}-R_{k}} \sum_{j=k+1}^{t_{k}} p_{j} q_{t_{k}-j} \frac{1}{2^{j}} \sum_{v=0}^{j}\left(\begin{array}{l}
j \\
v
\end{array}\right) x_{v}=L ;
$$


and for every $0<\mathrm{t}<1$,

$$
s t-\lim _{k} \frac{1}{R_{k}-R_{t_{k}}} \sum_{j=t_{k}+1}^{k} p_{j} q_{k-j} \frac{1}{2^{j}} \sum_{v=0}^{j}\left(\begin{array}{l}
j \\
v
\end{array}\right) x_{v}=L .
$$

Proof. (I) Let us consider the case where $t>1$. Then we obtain

$$
\begin{aligned}
& \frac{1}{R_{t_{n}}-R_{n}} \sum_{k=n+1}^{t_{n}} p_{k} q_{t_{n}-k} \frac{1}{2^{k}} \sum_{v=0}^{k}\left(\begin{array}{l}
k \\
v
\end{array}\right)\left(x_{v}-L\right) \\
= & \frac{R_{t_{n}}}{R_{t_{n}}-R_{n}} \frac{1}{R_{t_{n}}} \sum_{k=0}^{t_{n}} p_{k} q_{t_{n}-k} \frac{1}{2^{k}} \sum_{v=0}^{k}\left(\begin{array}{l}
k \\
v
\end{array}\right)\left(x_{v}-L\right) \\
& -\frac{R_{n}}{R_{t_{n}}-R_{n}} \frac{1}{R_{n}} \sum_{k=0}^{n} p_{k} q_{n-k} \frac{1}{2^{k}} \sum_{v=0}^{k}\left(\begin{array}{l}
k \\
v
\end{array}\right)\left(x_{v}-L\right) \\
= & \frac{R_{t_{n}}}{R_{t_{n}}-R_{n}} \frac{1}{R_{t_{n}}} \sum_{k=0}^{t_{n}} p_{k} q_{t_{n}-k} \frac{1}{2^{k}} \sum_{v=0}^{k}\left(\begin{array}{l}
k \\
v
\end{array}\right)\left(x_{v}-L\right) \\
& -\frac{R_{t_{n}}}{R_{t_{n}}-R_{n}} \frac{1}{R_{t_{n}}} \sum_{k=0}^{n} p_{k}\left(q_{t_{n}-k}+q_{n-k}-q_{n-k}\right) \frac{1}{2^{k}} \sum_{v=0}^{k}\left(\begin{array}{l}
k \\
v
\end{array}\right)\left(x_{v}-L\right) \\
= & \frac{R_{t_{n}}}{R_{t_{n}}-R_{n}} \frac{1}{R_{t_{n}}} \sum_{k=0}^{t_{n}} p_{k} q_{t_{n}-k} \frac{1}{2^{k}} \sum_{v=0}^{k}\left(\begin{array}{l}
k \\
v
\end{array}\right)\left(x_{v}-L\right) \\
& -\frac{R_{n}}{R_{t_{n}}-R_{n}} \frac{1}{R_{n}} \sum_{k=0}^{n} p_{k} q_{n-k} \frac{1}{2^{k}} \sum_{v=0}^{k}\left(\begin{array}{l}
k \\
v
\end{array}\right)\left(x_{v}-L\right) \\
& -\frac{R_{n}}{R_{t_{n}}-R_{n}} \frac{1}{R_{n}} \sum_{k=0}^{n} p_{k}\left(q_{t_{n}-k}-q_{n-k}\right) \frac{1}{2^{k}} \sum_{v=0}^{k}\left(\begin{array}{l}
k \\
v
\end{array}\right)\left(x_{v}-L\right) .
\end{aligned}
$$

From

$$
\lim _{n} \sup \frac{R_{t_{n}}}{R_{t_{n}}-R_{n}}<\infty,
$$

definition of the sequence $\left(q_{n}\right)$, Lemma 2 and relation (17), we get relation (15).

(II) In this case we have that $0<t<1$. Then

$$
\frac{1}{R_{n}-R_{t_{n}}} \sum_{k=t_{n}+1}^{n} p_{k} q_{n-k} \frac{1}{2^{k}} \sum_{v=0}^{k}\left(\begin{array}{l}
k \\
v
\end{array}\right) x_{v}=\frac{R_{n}}{R_{n}-R_{t_{n}}} \frac{1}{R_{n}} \sum_{k=0}^{n} p_{k} q_{n-k} \frac{1}{2^{k}} \sum_{v=0}^{k}\left(\begin{array}{l}
k \\
v
\end{array}\right) x_{v}-
$$


$\frac{R_{t_{n}}}{R_{n}-R_{t_{n}}} \frac{1}{R_{t_{n}}} \sum_{k=0}^{t_{n}} p_{k} q_{n-k} \frac{1}{2^{k}} \sum_{v=0}^{k}\left(\begin{array}{l}k \\ v\end{array}\right) x_{v}=\frac{R_{t_{n}}}{R_{n}-R_{t_{n}}} \frac{1}{R_{t_{n}}} \sum_{k=0}^{n} p_{k} q_{n-k} \frac{1}{2^{k}} \sum_{v=0}^{k}\left(\begin{array}{l}k \\ v\end{array}\right) x_{v}-$ $\frac{R_{t_{n}}}{R_{n}-R_{t_{n}}} \frac{1}{R_{t_{n}}} \sum_{k=0}^{t_{n}} p_{k} q_{t_{n}-k} \frac{1}{2^{k}} \sum_{v=0}^{k}\left(\begin{array}{l}k \\ v\end{array}\right) x_{v}-\frac{1}{R_{n}-R_{t_{n}}} \sum_{k=0}^{t_{n}} p_{k}\left(q_{n-k}-q_{t_{n}-k}\right) \frac{1}{2^{k}} \sum_{v=0}^{k}\left(\begin{array}{l}k \\ v\end{array}\right) x_{v}$.

Now proof of the proposition is similar to the first part.

\section{Proofs of the theorems}

Proof of Theorem 3. Necessity. Suppose that $\lim _{n \rightarrow \infty} x_{n}=L$, and (6) holds. Following Proposition 1, we have

$$
\begin{aligned}
& s t-\lim _{n \rightarrow \infty} \frac{1}{R_{\lambda_{n}}-R_{n}} \sum_{k=n+1}^{\lambda_{n}} p_{k} q_{\lambda_{n}-k} \frac{1}{2^{k}} \sum_{v=0}^{k}\left(\begin{array}{l}
k \\
v
\end{array}\right)\left(x_{v}-x_{n}\right)= \\
& s t-\lim _{n \rightarrow \infty} \frac{1}{R_{\lambda_{n}}-R_{n}} \sum_{k=n+1}^{\lambda_{n}} p_{k} q_{\lambda_{n}-k} \frac{1}{2^{k}} \sum_{v=0}^{k}\left(\begin{array}{l}
k \\
v
\end{array}\right) x_{v}-x_{n}=0,
\end{aligned}
$$

for every $\lambda>1$. In case where $0<\lambda<1$, we find that

$$
\begin{aligned}
& s t-\lim _{n \rightarrow \infty} \frac{1}{R_{n}-R_{\lambda_{n}}} \sum_{k=\lambda_{n}+1}^{n} p_{k} q_{n-k} \frac{1}{2^{k}} \sum_{v=0}^{k}\left(\begin{array}{l}
k \\
v
\end{array}\right)\left(x_{n}-x_{v}\right)= \\
& x_{n}-s t-\lim _{n \rightarrow \infty} \frac{1}{R_{n}-R_{\lambda_{n}}} \sum_{k=\lambda_{n}+1}^{n} p_{k} q_{n-k} \frac{1}{2^{k}} \sum_{v=0}^{k}\left(\begin{array}{l}
k \\
v
\end{array}\right) x_{v}=0 .
\end{aligned}
$$

Sufficiency. Assume that conditions (7) and (8) are satisfied. In what follows we will prove that $s t-\lim _{n \rightarrow \infty} x_{n}=L$. Or equivalently, st- $\lim \left(N_{p, q}^{n} E_{n}^{1}-x_{n}\right)=$ 0 . First we will consider the case where $t>1$. We will start from this estimation:

$$
\begin{aligned}
x_{n} & -N_{p, q}^{n} E_{n}^{1} \\
= & \frac{R_{t_{n}}}{R_{t_{n}}-R_{n}}\left[\frac{1}{R_{t_{n}}} \sum_{j=0}^{t_{n}} p_{j} q_{t_{n}-j} \frac{1}{2^{j}} \sum_{v=0}^{j}\left(\begin{array}{l}
j \\
v
\end{array}\right) x_{v}-\frac{1}{R_{n}} \sum_{j=0}^{n} p_{j} q_{n-j} \frac{1}{2^{j}} \sum_{v=0}^{j}\left(\begin{array}{l}
j \\
v
\end{array}\right) x_{v}\right] \\
& -\frac{1}{R_{t_{n}}-R_{n}} \sum_{j=n+1}^{t_{n}} p_{j} q_{t_{n}-j} \frac{1}{2^{j}} \sum_{v=0}^{j}\left(\begin{array}{l}
j \\
v
\end{array}\right)\left(x_{v}-x_{n}\right) .
\end{aligned}
$$


For any $\epsilon>0$, we obtain:

$$
\begin{gathered}
\left\{k \leq R_{n}: x_{k}-N_{p, q}^{n} E_{n}^{1} \geq \epsilon\right\} \subset\left\{k \leq R_{n}: \frac{R_{t_{k}}}{R_{t_{k}}-R_{k}}\left(N_{p, q}^{t_{k}} E_{k}^{1}-N_{p, q}^{k} E_{k}^{1}\right) \geq \frac{\epsilon}{2}\right\} U \\
\left\{k \leq R_{n}: \frac{1}{R_{t_{k}}-R_{k}} \sum_{j=k+1}^{t_{k}} p_{j} q_{t_{k}-j} \frac{1}{2^{j}} \sum_{v=0}^{j}\left(\begin{array}{l}
j \\
v
\end{array}\right)\left(x_{v}-x_{k}\right) \leq-\frac{\epsilon}{2}\right\} .
\end{gathered}
$$

From relation (7), it follows that for every $\gamma>0$, exists a $t>1$ such that

$$
\limsup _{n} \frac{1}{R_{n}}\left|\left\{k \leq R_{n}: \frac{1}{R_{t_{k}}-R_{k}} \sum_{j=k+1}^{t_{k}} p_{j} q_{t_{k}-j} \frac{1}{2^{j}} \sum_{v=0}^{j}\left(\begin{array}{l}
j \\
v
\end{array}\right)\left(x_{v}-x_{k}\right) \leq-\epsilon\right\}\right| \leq \gamma .
$$

By Lemma 2 and relation (18) we get

$$
\limsup _{n} \frac{1}{R_{n}}\left|\left\{k \leq R_{n}:\left|R_{t_{k}}\left(R_{t_{k}}-R_{k}\right)^{-1}\left(N_{p, q}^{t_{k}} E_{k}^{1}-N_{p, q}^{k} E_{k}^{1}\right)\right| \geq \frac{\epsilon}{2}\right\}\right|=0 .
$$

Combining last three relations we have:

$$
\limsup _{n} \frac{1}{R_{n}}\left|\left\{k \leq R_{n}: x_{k}-N_{p, q}^{k} E_{k}^{1} \geq \epsilon\right\}\right| \leq \gamma,
$$

and $\gamma$ is arbitrary, we conclude that for every $\epsilon>0$,

$$
\limsup _{n} \frac{1}{R_{n}}\left|\left\{k \leq R_{n}: x_{k}-N_{p, q}^{k} E_{k}^{1} \geq \epsilon\right\}\right|=0 .
$$

Now we consider case where $0<t<1$. From above we get that:

$$
\begin{aligned}
x_{n}- & N_{p, q}^{n} E_{n}^{1} \\
= & \frac{R_{t_{n}}}{R_{n}-R_{t_{n}}}\left[\frac{1}{R_{n}} \sum_{j=0}^{n} p_{j} q_{n-j} \frac{1}{2^{j}} \sum_{v=0}^{j}\left(\begin{array}{l}
j \\
v
\end{array}\right) x_{v}-\frac{1}{R_{t_{n}}} \sum_{j=0}^{t_{n}} p_{j} q_{t_{n}-j} \frac{1}{2^{j}} \sum_{v=0}^{j}\left(\begin{array}{l}
j \\
v
\end{array}\right) x_{v}\right] \\
& +\frac{1}{R_{n}-R_{t_{n}}} \sum_{j=t_{n}+1}^{n} p_{j} q_{n-j} \frac{1}{2^{j}} \sum_{v=0}^{j}\left(\begin{array}{l}
j \\
v
\end{array}\right)\left(x_{n}-x_{v}\right) .
\end{aligned}
$$

For any $\epsilon>0$,

$$
\left\{k \leq R_{n}: x_{k}-N_{p, q}^{k} E_{k}^{1} \geq \epsilon\right\} \subset\left\{k \leq R_{n}: \frac{R_{t_{k}}}{R_{k}-R_{t_{k}}}\left(N_{p, q}^{k} E_{k}^{1}-N_{p, q}^{t_{k}} E_{k}^{1}\right) \geq \frac{\epsilon}{2}\right\} U
$$




$$
\left\{k \leq R_{n}: \frac{1}{R_{k}-R_{t_{k}}} \sum_{j=t_{k}+1}^{k} p_{j} q_{k-j} \frac{1}{2^{j}} \sum_{v=0}^{j}\left(\begin{array}{l}
j \\
v
\end{array}\right)\left(x_{k}-x_{v}\right) \leq-\frac{\epsilon}{2}\right\} .
$$

For same reasons as in the case where $t>1$, by Lemma 2 , we have that for every $\epsilon>0$,

$$
\lim \sup _{n} \frac{1}{R_{n}}\left|\left\{k \leq R_{n}: x_{k}-N_{p, q}^{k} E_{k}^{1} \leq-\epsilon\right\}\right|=0
$$

Finally from relations (19) and (20) we get:

$$
\lim \sup _{n} \frac{1}{R_{n}}\left|\left\{k \leq R_{n}:\left|x_{k}-N_{p, q}^{k} E_{k}^{1}\right| \geq \epsilon\right\}\right|=0 .
$$

Remark 2 Let us suppose that $\mathrm{st}-\lim _{\mathrm{k}} \mathrm{x}_{\mathrm{k}}=\mathrm{L}$, st $-\lim _{\mathrm{k}} \mathrm{N}_{\mathrm{p}, \mathrm{q}}^{\mathrm{k}} \mathrm{E}_{\mathrm{k}}^{1}=\mathrm{L}$ and relation (6) satisfies. Then for every $\mathrm{t}>1$, relation (9) holds, and in case where $0<\mathrm{t}<1$, relation (10) is valid.

Proof of Theorem 4. We omit it because it is similar to the Theorem 3 .

\section{References}

[1] D. Borwein, On products of sequences, J. London Math. Soc. , 33 (1958), $352-357$.

[2] N. L. Braha, Tauberian conditions under which $\lambda$-statistical convergence follows from statistical summability $(\mathrm{V}, \lambda)$, Miskolc Math. Notes, 16 (2) (2015), 695-703.

[3] N. L. Braha and Ismet Temaj, Tauberian conditions under which statistical convergence follows from statistical summability $(E C)_{1}^{n}$, Bol. Soc. Parana. Mat., (3) 37 (2019), no. 4, 9-17.

[4] N. L. Braha, Tauberian theorems under Nörlund-Cesáro summability methods (357-411), Current Topics in Summability Theory and Applications, editors, Hemen Dutta and Billy E. Rhoades, Springer, 2016.

[5] Ibrahim Çanak, Naim L. Braha and Ümit Totur, A Tauberian theorem for the generalized Nörlund summability method, Georgian Journal of Mathematics (article in press). 
[6] R. Kiesel, General Nörlund transforms and power series methods, Math. Z., 214 (2) (1993), 273-286.

[7] R. Kiesel, U. Stadtmüller, Tauberian- and convexity theorems for certain (N, p, q)-means, Canad. J. Math., 46 (5) (1994), 982-994.

[8] Valdete Loku and Naim L. Braha, Tauberian theorems by weighted summability method, Armen. J. Math., 9 (1) (2017), 35-42.

[9] U. Stadtmüller and A. Tali, On certain families of generalized Nörlund methods and power series methods, J. Math. Anal. Appl., 238 (1) (1999), $44-66$.

Received: October 15, 2018 